\title{
Scheduling Finite Difference Approximations for DAG-Modeled Large Scale Applications
}

\author{
Xavier Meyer \\ Swiss Institute of Bioinformatics \\ University of Lausanne \\ Department of Ecology and Evolution \\ Lausanne 1015, Switzerland \\ xavier.meyer.2@unil.ch
}

\author{
Bastien Chopard \\ Swiss Institute of Bioinformatics \\ University of Geneva \\ Department of Computer Science \\ Geneva 1211, Switzerland
}

\author{
Nicolas Salamin \\ Swiss Institute of Bioinformatics \\ University of Lausanne \\ Department of Ecology and Evolution \\ Lausanne 1015, Switzerland
}

\begin{abstract}
An increasing number of scientific domains are confronted with the arduous task of managing large scale applications. For such applications, gradient estimations come at a large computational cost. Despite notable advances in automatic differentiation during the last years, its use in this context may reveal too costly in memory, inadequate for parallel architecture or require expert knowledge. For these reasons, we investigate an alternative approach that uses the finite difference method to evaluate the gradient of functions modeled as a directed acyclic graph. This approach enables the reuse of partial results from previous partial derivatives evaluations and thus reduces the computational cost.

We identify a discrete optimization problem arising in the limitedmemory context of large scale applications that aims to maximize the computational efficiency of the gradient approximation by scheduling the partial derivatives. This optimization problem is extended to consider the partitioning of the computations on multiple processors. We further derive some properties of these optimization problems, such as their upper bound on performance gains.

Following a brief description of algorithms designed to obtain sensible solutions for both problems, we study the increase in performance resulting from sequential and parallel schedules obtained for synthetic DAGs. Finally, we employ this approach to accelerate the gradient evaluation of DAGs representing real evolutionary biology models. For one of these large scale applications, our approach is shown to be nearly 400 times faster than a state-of-the-art software in sequential, and more than 11,000 times faster when using 256 processors.
\end{abstract}

\section{CCS CONCEPTS}

- Computing methodologies $\rightarrow$ Parallel algorithms; • Mathematics of computing $\rightarrow$ Numerical differentiation; Combinatoric problems; Combinatorial optimization; • Theory of computation $\rightarrow$ Parallel computing models; Evolutionary algorithms; - Applied computing $\rightarrow$ Molecular sequence analysis;

Permission to make digital or hard copies of all or part of this work for personal or classroom use is granted without fee provided that copies are not made or distributed for profit or commercial advantage and that copies bear this notice and the full citation on the first page. Copyrights for components of this work owned by others than the author(s) must be honored. Abstracting with credit is permitted. To copy otherwise, or republish, to post on servers or to redistribute to lists, requires prior specific permission and/or a fee. Request permissions from permissions@acm.org.

PASC '17, fune 26-28, 2017, Lugano, Switzerland

() 2017 Copyright held by the owner/author(s). Publication rights licensed to Association for Computing Machinery.

ACM ISBN 978-1-4503-5062-4/17/06 . \$ \$15.00

https://doi.org/10.1145/3093172.3093231

\section{ACM Reference format:}

Xavier Meyer, Bastien Chopard, and Nicolas Salamin. 2017. Scheduling Finite Difference Approximations for DAG-Modeled Large Scale Applications. In Proceedings of PASC '17, Lugano, Switzerland, June 26-28, 2017, 12 pages. https://doi.org/10.1145/3093172.3093231

\section{INTRODUCTION}

Many applications in science require the efficient evaluation of gradients. These applications range from finance [50] to biology [9] and also include all forms of gradient-based optimization methods [29]. As we enter the Big Data era [17, 43], the available amount of data enables more and more complex applications to be considered. Efficiently computing gradients for such large scale applications represents however a growing challenge due to their ever increasing computational cost and memory requirement.

One typical example of such application arises in the field of evolutionary biology. The recent surge in molecular data due to the advances in DNA sequencing [25] enabled previously unchallenged hypotheses to be investigated. One of such hypotheses is Darwin's theory of positive selection which is nowadays examined using statistical analysis [28]. Optimization methods employed to this end require numerous costly gradient evaluations and thus subsequent efforts were necessary to obtain an efficient implementation of the statistical model $[45,51,52]$. However, such implementations still struggle to analyse, in an reasonable time, nowadays large-scale molecular datasets that comprise several thousands of species.

For such applications where numerical methods must be employed to evaluate the gradient, two different computational approaches are available. The first one, the method of finite difference (FDM), estimates the gradient by observing the change in the function output in response to small perturbations of its input variables. This method is straightforward to implement and can benefit from parallel architectures [46]. However, this method is rarely employed for large scale applications given that its computational cost grows linearly with the number of input variables [24].

The second method, automatic differentiation (AD), breaks down the elementary arithmetic operations of the algorithm representing the function to apply the chain rule [5]. An important advantage of this method is its improved accuracy when compared to the FDM; however, to benefit from this improved accuracy, the original algorithm must be altered to evaluate the partial derivative of all its elementary arithmetic operations. Specialized tools can be used to automate the generation of the $\mathrm{AD}$ code by using source code transformation [15] or operator overload [49]. However, to our knowledge, such tools still have troubles when dealing with parallel 
algorithm as noted by Rasch et al. [38]: "no current automatic differentiation tool is capable of handling message-passing parallel programs in a fully automated way".

Two main variants of $\mathrm{AD}$ exist: the forward and reverse modes. The forward mode shares the same complexity as FDM [37] and is relatively easily adapted to parallel architectures once the forward AD code is available. The second variant, the reverse mode, is particularly efficient for gradient evaluations [26]. While having a constant complexity, this approach suffers from two limitations for large scale computations: its memory footprint grows proportionally with the number of elementary operations in the algorithm and, more importantly, its joint use with functions implemented on distributed architecture is difficult [14].

These memory issues can be addressed by using advanced strategies known as checkpointing that relax the memory requirements at the cost of additional computations [13, 47]. However, the elaboration of specialized tool producing the reverse mode $\mathrm{AD}$ code for distributed computing remains an open question $[27,44]$ that increase in complexity when checkpointing is taken into consideration [16]. Successful use of distributed architectures has been limited to proofs of concept for AD tools [42] or required significant implementation efforts $[8,21]$.

In this article, we investigate an alternative, and simple, approach based on the FDM aiming at efficiently evaluating the gradients of large-scale applications. We consider applications for which the functions are already modeled as directed acyclic graphs (DAG) where vertices represent computations and edges identifies data dependencies. This generic representation of computations has already proved its usefulness in various applications such as the scheduling of parallel computations $[1,23]$ or the formalisation of statistical models as graphical models $[19,20]$. In the context of gradient evaluations, it enables the re-use of previously computed partial results: this strategy akin to belief-propagation in graphical models [48] can be seen as a form of checkpointing [7].

More precisely, we investigate the discrete optimization problem aiming to maximize the computational efficiency that arises from the scheduling of the perturbations applied for the FDM in a memory-limited environment. In order to benefit from parallel architectures, we then extend this optimization problem to schedule and partition these perturbations across multiple processors. Then, the key concepts required to implement genetic algorithms that obtain good solutions for these optimization problems are described. Finally, using these algorithms, we analyze the potential gains resulting from the sequential and parallel scheduling of the perturbations on synthetic DAG as well as on DAGs representing the computations of two existing evolutionary biology models.

\section{PRELIMINARIES}

The problem considered is the computation of the gradient $\nabla f$ of a smooth function $f: \mathbb{R}^{d} \rightarrow \mathbb{R}$ using the FDM. The forwarddifference approximation of partial derivatives is defined as

$$
\frac{\partial f}{\partial x_{i}}(X) \approx \frac{f\left(X+\epsilon u_{i}\right)-f(X)}{\epsilon}
$$

where $X \in \mathbb{R}^{d}, \epsilon$ being a small positive scalar and $u_{i}$ the $i$ th unit vector. The vector $\epsilon u_{i}$ represents thus a small perturbation to $x_{i}$, the $i$ th element of $X$.

The functions $f$ considered are decomposable as directed acyclic graph (DAG) defined as $G=(V, E)$. Nodes represent partial computations of $f$ and edges $e=\left(v_{s}, v_{t}\right)$ express the data dependency of parent node $v_{s}$ for a partial result computed by its child node $v_{t}$. The computational cost of each node is defined by a function $c: v \in V \rightarrow \mathbb{R}_{+}$. This cost function is either known a priori, estimated by measuring nodes execution time or simplified as $c: v \in V \rightarrow 1$. Finally, an input variable $x_{i} \in X$ acts as a terminal node $v$, having thus no children and at least one parent.

Assuming that the partial results identifying data dependencies are memorized locally on each node after the last function evaluation $f(X)$, an increase of $\alpha$ in a single variable $x_{i}$ requiring the evaluation of $f\left(X+\alpha u_{i}\right)$ induces then solely a partial computation of the DAG. Indeed, only the set of nodes $\Psi_{\{i\}}$ containing all the ancestral nodes of the terminal node $v$ associated with variable $x_{i}$ must be recomputed in order to evaluate $f\left(X+\alpha u_{i}\right)$.

Definition 2.1. A change in value of a parameter $x_{i}$ always induces computations, therefore

$$
\forall i \in(1 . . m), \Psi_{i} \neq \emptyset
$$

The notation $\Psi_{i}$ is equivalent to $\Psi_{\{i\}}$.

Definition 2.2. The set of vertices $\Psi_{\mathcal{I}}$ is equivalent to the union of the set of vertices induced by change of single parameters $\left\{x_{i}: i \in \mathcal{I}\right\} \subset$ $X$ such that

$$
\Psi_{\mathcal{I}}=\bigcup_{i \in \mathcal{I}} \Psi_{i}
$$

Definition 2.3. The cost function $C$ acts as a weighted count of the set $\Psi_{\mathcal{I}}$ in function of vertex weights $c(v)>0: v \in \Psi_{\mathcal{I}}$, as

$$
C\left(\Psi_{\mathcal{I}}\right)=\sum_{v \in \Psi_{I}} c(v) \rightarrow C\left(\Psi_{I}\right)>0 .
$$

By extension this counting function follows the principle of inclusionexclusion, implying that the cost of a set $\mathcal{I}=\{a, b\}$ can be decomposed as

$C\left(\Psi_{\mathcal{I}}\right)=\mathcal{C}\left(\Psi_{\{a, b\}}\right)=\mathcal{C}\left(\Psi_{a} \cup \Psi_{b}\right)=C\left(\Psi_{a}\right)+C\left(\Psi_{b}\right)-C\left(\Psi_{a} \cap \Psi_{b}\right)$.

Definition 2.4. Given that $\left(\Psi_{a} \cap \Psi_{b}\right) \subseteq \Psi_{a}$, and $\left(\Psi_{a} \cap \Psi_{b}\right) \subseteq \Psi_{b}$, the cost $C$ of the intersection of two sets cannot exceed the cost of one of the sets, such that

$$
C\left(\Psi_{a} \cap \Psi_{b}\right) \leq C\left(\Psi_{a}\right) \quad \text { and } \quad C\left(\Psi_{a} \cap \Psi_{b}\right) \leq C\left(\Psi_{b}\right) .
$$

\section{OPTIMIZING THE EFFICIENCY OF GRADIENT EVALUATIONS}

In order to evaluate $\nabla f(X)$ using Eq. (1), the response to perturbations $\epsilon u_{i}$, for each $x_{i} \in X$, forming the sequence defined as

$$
\mathcal{S}=\left\{f(X), f\left(X+\epsilon u_{1}\right), . ., f\left(X+\epsilon u_{d}\right)\right\},
$$

must be evaluated. Unfortunately, the computational cost represented by this sequence grows linearly with $d$ given that $d+1$ function evaluations are required. This cost can however be reduced by maintaining a copy in memory of the complete DAG data dependencies as checkpoint after the evaluation of $f(X)$. Indeed, 
this checkpoint can be employed to reset the state of the DAG prior to each perturbation evaluation $\epsilon \mathcal{u}_{i}$. Such evaluation would then solely require a partial computation defined by the set of nodes $\Psi_{i}$. While this approach can increase the efficiency of the gradient evaluations, it may reveal infeasible in the memory-limited context of large scale applications due to the expensive memory cost of maintaining a second copy of the complete set of data dependencies.

In order to still benefit from partial computations without increasing the memory footprint, the computations sequence of Eq. (2) can be reformulated by assuming an arbitrary order $\mathcal{R}=\left(r_{1}, . ., r_{i}, . ., r_{d}\right)$ of the perturbations $\epsilon u_{i}$. These function evaluations can then be defined as the sequence

$$
\mathcal{S}_{\text {eval }}=\left(f\left(X_{0}\right), f\left(X_{1}\right), \ldots, f\left(X_{i}\right), . ., f\left(X_{d}\right)\right)
$$

with

$$
\begin{array}{r}
X_{0}=X, \\
X_{1}=X_{0}+\epsilon u_{r_{1}}, \\
X_{2}=X_{1}-\epsilon u_{r_{1}}+\epsilon u_{r_{2}}, \\
X_{i}=X_{(i-1)}-\epsilon u_{r_{i-1}}+\epsilon u_{r_{i}}, \\
X_{d}=X_{(d-1)}-\epsilon u_{r_{d-1}}+\epsilon u_{r_{d}} .
\end{array}
$$

Each function evaluations $f\left(X_{i}\right)$ with $i>1$ of $\mathcal{S}$ represents thus a partial computation identified by the set of nodes $\Psi_{\left\{r_{i-1}, r_{i}\right\}}$.

The ordering $\mathcal{R}$ of the perturbations plays a key role by defining the partial computations induced by the sequence of function evaluations $\mathcal{S}_{\text {eval }}$. The impact of $\mathcal{R}$ clearly appears when the sequence $\mathcal{S}_{\text {eval }}$ is expressed such as to represent the sequence of nodes sets required to evaluate the gradient:

$$
\mathcal{S}_{\text {nodes }}=\left(V, \Psi_{r_{1}}, . ., \Psi_{\left\{r_{i-1}, r_{i}\right\}}, . ., \Psi_{\left\{r_{d-1}, r_{d}\right\}}\right) .
$$

Therefore, the order of permutation $\mathcal{R}$ also defines the cost of each function evaluation $f\left(X_{i}\right)$. Indeed, the partial computation identified by the nodes set $\Psi_{\left\{r_{i-1}, r_{i}\right\}}$ may be significantly different in function of its two composing subsets $\Psi_{r_{i-1}}$ and $\Psi_{r_{i}}$ (Def. 2.2).

\subsection{Characterizing the impact of ordering $\mathcal{R}$}

The cost induced by a partial computation is defined as

$$
\mathcal{C}\left(\Psi_{\left\{r_{i-1}, r_{i}\right\}}\right)=\mathcal{C}\left(\Psi_{r_{i-1}} \cup \Psi_{r_{i}}\right)=\mathcal{C}\left(\Psi_{r_{i-1}}\right)+C\left(\Psi_{r_{i}} \backslash \Psi_{r_{i-1}}\right) .
$$

This cost is therefore of at least $C\left(\Psi_{r_{i-1}}\right)$. The additional $\operatorname{cost} C\left(\Psi_{r_{i}} \backslash \Psi_{r_{i-1}}\right)$ variates in function of the perturbation paired. In the best case, both nodes sets solely differ from a single node $v_{r_{i-1}}$ and thus $C\left(\Psi_{r_{i}} \backslash \Psi_{r_{i-1}}\right)=C\left(\left\{v_{r_{i-1}}\right\}\right)$. Oppositely, in the worst case, both nodes sets have only in common the root node of the DAG $v_{R}$ and thus $C\left(\Psi_{r_{i}} \backslash \Psi_{r_{i-1}}\right)=C\left(\Psi_{r_{i-1}} \backslash v_{R}\right)$.

Therefore, the best case for the computation of the sequence of nodes defined in Eq. (5) is given by

$$
C_{b}=C(V)+C\left(\Psi_{r_{1}}\right)+\sum_{i=2}^{d}\left[C\left(\Psi_{r_{i}}\right)+C\left(v_{r_{i}}\right)\right] .
$$

Assuming that $C\left(\Psi_{r_{i}}\right) \gg C\left(v_{r_{i}}\right)$ then this equation simplifies to

$$
C_{b} \approx C(V)+\sum_{i=1}^{d}\left[C\left(\Psi_{r_{i}}\right)\right]
$$

In the worst case, the computational cost is given by

$$
C_{w}=C(V)+C\left(\Psi_{r_{1}}\right)+\sum_{i=2}^{d}\left[C\left(\Psi_{r_{i}}\right)+C\left(\Psi_{r_{i-1}}\right)-C\left(v_{R}\right)\right] .
$$

Similarly, under the assumption that the computational cost of the root node $C\left(v_{R}\right)$ is strictly smaller than the cost of the two partial updates then the equation simplifies to

$$
C_{w} \approx C(V)+2 \sum_{i=1}^{d-1}\left[C\left(\Psi_{r_{i}}\right)\right]+C\left(\Psi_{r_{d}}\right)
$$

The maximal impact of the ordering $\mathcal{R}$ can then be characterized by the slowdown induced by the worst possible ordering when compared to the best one. This slowdown is defined as

$$
\frac{C_{w}}{C_{b}}=\frac{C(V)+2 \sum_{i=1}^{d-1}\left[C\left(\Psi_{r_{i}}\right)\right]+C\left(\Psi_{r_{d}}\right)}{C(V)+\sum_{i=1}^{d}\left[C\left(\Psi_{r_{i}}\right)\right]}
$$

For highly dimensional function $(d \rightarrow \infty)$ it simplifies to

$$
\lim _{d \rightarrow \infty} \frac{C_{w}}{C_{b}} \approx \frac{C(V)+2 \sum_{i=1}^{\infty}\left[C\left(\Psi_{r_{i}}\right)\right]}{C(V)+\sum_{i=1}^{\infty}\left[C\left(\Psi_{r_{i}}\right)\right]} .
$$

The worst slowdown occurs when the overall cost of partial computations cost dominates $C(V)$. Under these assumptions, the ordering $\mathcal{R}$ could induce 2 -fold slower gradient evaluations. However, if $C(V)$ is not dominated, the slowdown is bounded to $3 / 2$ since the overall cost of partial computation cannot be lower than $C(V)$.

Obtaining a good ordering $\mathcal{R}$ is therefore important given that it can lead to twice faster gradient evaluations when compared to the worst possible ordering. While such performance gain may seem limited, it is important to note that this is a multiplicative gain on the performance increase already achieved by using the sequence of partial computations defined in Eq. (3).

\subsection{Optimizing the sequential ordering}

In order to reduce the computational cost of $\nabla f$, the best ordering $\mathcal{R}$ among the $(\mathcal{R})_{d}^{d}=d$ ! possible permutations of the perturbations must be found. This problem amount to find $\mathcal{R}$ such that

$$
\min _{\mathcal{R}} C(V)+C\left(\Psi_{r_{1}}\right)+\sum_{i=2}^{d} C\left(\Psi_{r_{i}}, \Psi_{r_{i-1}}\right) .
$$

By reformulating the first term $C\left(\Psi_{r_{1}}\right)$ as $C\left(\Psi_{r_{0}}, \Psi_{r_{1}}\right)$ with $\Psi_{r_{0}}=$ $\emptyset$, this problem consists in finding the ordered arrangement of perturbations minimizing the pairwise partial computations cost. This optimization problem is equivalent to the well-known travelling salesman problem (TSP [4]) without return to the initial city.

Indeed, the TSP aims to find the tour visiting all cities that minimizes the overall distance travelled. The minimization problem of Eq. (12) similarly aims to find the tour evaluating all perturbations, only once, such as to minimizes the computational cost defined by the pairwise cost function $C\left(\Psi_{r_{i}}, \Psi_{r_{i+1}}\right)$. This cost function, or distance between two perturbations, defined for this peculiar instance of the TSP enforces the strict triangle inequality property. 


\subsubsection{Proof of strict triangle inequality.}

THEOREM 3.1. The distance, or cost $C$, between a pair of perturbations used to define the instance of the traveling salesman problem (Eq. (12)) is subject to the strict triangle inequality defining that

$$
C\left(\Psi_{\{a, c\}}\right)<C\left(\Psi_{\{a, b\}}\right)+C\left(\Psi_{\{b, c\}}\right)
$$

with $a, b, c$ representing the index $i \in(1 . . d)$ of the perturbations $u_{i} \epsilon$ and $\Psi_{i}$ representing the set of vertices in the directed task graph that must be recomputed following a perturbation of parameter $x_{i}$.

Proof. Starting from the strict triangle inequality defined as

$$
C\left(\Psi_{\{a, c\}}\right)<C\left(\Psi_{\{a, b\}}\right)+C\left(\Psi_{\{b, c\}}\right) .
$$

The cost function can be decomposed in function of the inclusionexclusion principle as,

$$
\begin{array}{r}
C\left(\Psi_{a}\right)+C\left(\Psi_{c}\right)-C\left(\Psi_{a} \cap \Psi_{c}\right)<C\left(\Psi_{a}\right)+C\left(\Psi_{b}\right)-C\left(\Psi_{a} \cap \Psi_{b}\right) \\
+C\left(\Psi_{b}\right)+C\left(\Psi_{c}\right)-C\left(\Psi_{b} \cap \Psi_{c}\right), \\
-C\left(\Psi_{a} \cap \Psi_{c}\right)<2 \times C\left(\Psi_{b}\right)-\left[C\left(\Psi_{a} \cap \Psi_{b}\right)+C\left(\Psi_{b} \cap \Psi_{c}\right)\right], \\
C\left(\Psi_{a} \cap \Psi_{b}\right)+C\left(\Psi_{b} \cap \Psi_{c}\right)<2 \times C\left(\Psi_{b}\right)+C\left(\Psi_{a} \cap \Psi_{c}\right) .
\end{array}
$$

Given that $C(\cdot) \geq 0$ and $C\left(\Psi_{b}\right)>0$ (Def. 2.1), the left hand side must at least be equal to $2 \times C\left(\Psi_{b}\right)$ to prove that the strict triangular inequality does not hold. Furthermore, the costs related to the set intersection $\left(\Psi_{a} \cap \Psi_{b}\right)$ or $\left(\Psi_{b} \cap \Psi_{c}\right)$ cannot exceed this cost $C\left(\Psi_{b}\right)$ (Def. 2.4). Therefore two cases are identified.

First case: At least one of the intersections $\left(\Psi_{a} \cap \Psi_{b}\right)$ or $\left(\Psi_{b} \cap \Psi_{c}\right)$ is strictly included in $\Psi_{b}$, such that

$$
\Downarrow
$$

$$
C\left(\Psi_{a} \cap \Psi_{b}\right)<C\left(\Psi_{b}\right) \text { or } C\left(\Psi_{b} \cap \Psi_{c}\right)<C\left(\Psi_{b}\right)
$$

$$
C\left(\Psi_{a} \cap \Psi_{b}\right)+C\left(\Psi_{b} \cap \Psi_{c}\right)<2 \times C\left(\Psi_{b}\right) .
$$

Therefore Eq. (13) holds and the strict triangle inequality is proven even if $C\left(\Psi_{a} \cap \Psi_{c}\right)=0$.

Second case: Both sets of intersections $\left(\Psi_{a} \cap \Psi_{b}\right)$ and $\left(\Psi_{b} \cap \Psi_{c}\right)$ are equal to $\Psi_{b}$, such that $C\left(\Psi_{a} \cap \Psi_{b}\right)=C\left(\Psi_{b} \cap \Psi_{c}\right)=C\left(\Psi_{b}\right)$. Therefore Eq. (13) can be reformulated as

$$
\begin{array}{r}
2 \times C\left(\Psi_{b}\right)<2 \times C\left(\Psi_{b}\right)+C\left(\Psi_{a} \cap \Psi_{c}\right), \\
0<C\left(\Psi_{a} \cap \Psi_{c}\right) .
\end{array}
$$

Given that $\Psi_{b} \subseteq \Psi_{a}$ and $\Psi_{b} \subseteq \Psi_{c}$, then $\Psi_{b} \subseteq\left(\Psi_{a} \cap \Psi_{c}\right)$ such that

$$
0<C\left(\Psi_{b}\right) \leq C\left(\Psi_{a} \cap \Psi_{c}\right) .
$$

Therefore, in this second case the strict triangle inequality also holds.

\subsection{Optimizing the parallel ordering}

To further reduce the time required to evaluate $\nabla f$, the function evaluations defined in Eq. (2) can be partitioned over $P$ processors given that the perturbations evaluations are independent. However, the partitioning of these $|\mathcal{S}|$ tasks remains a challenging problem.

The use of partial computations induces highly variable tasks costs. Therefore, these tasks must be partitioned such as to balance the amount of work assigned to each processor. This NPComplete problem, well known as the independent tasks scheduling problem [41], has been studied for decades and several heuristic approaches having an affordable complexity of $O(n \log (n))$ have been designed to produce good tasks schedules.

These approaches are however unusable in this peculiar situation given that the cost of a task is dependent of its neighboring tasks. Indeed, while the tasks are independent with respect to their inputs and outputs, their computational cost is dependent of their ordering as illustrated in Eq. (3). Moving a task from a partition to another impacts therefore several costs. First, removing a task from a partition changes the cost of the next partial computation of the sequence. Second, inserting this same task in another partition updates the cost of the task in question as well as its following task.

Therefore, the difficulty of scheduling the sequence of perturbation evaluations $\mathcal{S}$ on $P$ processors comes from the partitioning $\mathcal{D}$ of the computations composing $\mathcal{S}$ in multiple subsets that must each be optimally ordered such that the parallel computational cost is minimized. The partitioning $\mathcal{D}$ in $P$ subsets combined with the subset permutations $\mathcal{R}^{(p)}$ is defined as

$$
\begin{aligned}
& \mathcal{D}=\left\{\mathcal{R}^{(p)}: p \in(1, \ldots, P)\right\} \\
& \text { subject to } \quad \emptyset \notin \mathcal{D}, \\
& \bigcup_{p \in(1, \ldots, P)} \mathcal{R}^{(p)}=\mathcal{R}, \\
& \bigcap_{p \in(1, \ldots, P)} \mathcal{R}^{(p)}=\emptyset .
\end{aligned}
$$

The cost of partition $R^{(p)}$ assigned to processor $p$ is defined as

$$
C\left(R^{(p)}\right)=\left\{\begin{array}{l}
C(V)+C\left(\Psi_{r_{1}^{(p)}}\right)+\sum_{i=2}^{\left|R^{(p)}\right|} C\left(\Psi_{r_{i}^{(p)}, r_{i-1}^{(p)}}\right) \text { if } p=1, \\
C\left(V \cup \Psi_{r_{1}^{(p)}}\right)+\sum_{i=2}^{\left|R^{(p)}\right|} C\left(\Psi_{r_{i}^{(p)}, r_{i-1}^{(p)}}\right) \text { if } p>1 .
\end{array}\right.
$$

The processor $p=1$ is designated to evaluate $f(X)$ at a $C(V)$ cost, while the others start directly by computing a perturbation. Since the initial state of the DAG is not up to date relatively to the evaluation $f(X)$, all nodes must initially be recomputed before the first perturbation evaluation is accomplished. While being extremely costly, this operation can be merged with the first perturbation and has a cost $C\left(V \cup \Psi_{r_{1}^{(p)}}\right)=C(V)$.

The total cost of a partitioning $\mathcal{D}$ is then given as

$$
C(\mathcal{D})=\sum_{p=1}^{P} C\left(\mathcal{R}^{(p)}\right) .
$$

Minimizing this total cost is closely related to the multiple Travelling Salesman Problem [6] which is himself a special case of the more complex Vehicle Routing Problem (VRP [22]).

While minimizing the overall computational cost is rather important, the aim of assigning the likelihood evaluations of $\mathcal{S}$ to different processors is to reduce the effective time required to evaluate all functions in $\mathcal{S}$. This time, for a computation involving $P$ processors, is identified by the time taken by the processor having the more work and thus being the last to finish its computation ${ }^{1}$. Therefore, the optimal tasks partitioning $\mathcal{D}$ and orderings $\mathcal{R}^{(p)}$ for a given number of processors $P$ is defined by

\footnotetext{
${ }^{1}$ Assuming an heterogeneous parallel architecture.
} 


$$
\underset{\mathcal{R}^{(\cdot)}, \mathcal{D}}{\arg \min }\left[\max _{p \in P}\left(\mathcal{C}\left(\mathcal{R}^{(p)}\right)\right)\right] .
$$

3.3.1 Upper bound of the parallel scheduling performance gain. The performance gain resulting from the use of parallel computing resources is bounded by the irreducible cost $C(V)$ induced by the initialization of the DAG (Eq. (14)). Indeed, assuming an optimal partitioning of the computations, the parallel work attributed to each processor can be approximated as

$$
C\left(R^{(\cdot)}\right)=C(V)+\frac{C(\mathcal{R})-C(V)}{P},
$$

where $\mathcal{C}(\mathcal{R})$ represents the sequential cost for the ordering $\mathcal{R}$. The first term identifies the irreducible cost of the initialization. The second one characterizes the cost of the remaining computations when being ideally balanced over the $p$ processors.

While this approximation is optimistic with respect to the load balance of the partitions, it is pessimistic with respect to their cost. Indeed, the strict triangle inequality property (Thm. 3.1) holds for the cost function and thus the partitioning of the sequential computations should result in a decrease in computational cost.

This approximation offers however a good basis to discuss the potential expectation and limitation of the speedup resulting from the use of multiple processors. Assuming a negligible communication cost between the processors, the speedup can be approximated as $\frac{C(\mathcal{R})}{C\left(\mathcal{R}^{(\cdot)}\right)}$ and the upper bound for the speedup is given as

$$
\lim _{P \rightarrow \infty} \frac{C(\mathcal{R})}{C\left(\mathcal{R}^{(\cdot)}\right)}=\lim _{P \rightarrow \infty} \frac{C(\mathcal{R})}{C(V)+\frac{C(\mathcal{R})-C(V)}{P}}=\frac{C(\mathcal{R})}{C(V)} .
$$

Therefore, the speedup is bounded by the amount of inherent sequential work $C(V)$ that each processor must process. Additionally, this theoretical analysis of the speedup reveals that DAGs having an initialization cost $C(V)$ strictly smaller than the full sequential cost $C(R)$ are more prone to present significant performance gain when computed with multiple processors.

\section{OBTAINING GOOD SCHEDULES}

Defining a good sequential scheduling for the gradient evaluation according to the minimization problem defined in Eq. (12) is easily achieved using one of the numerous software dedicated to the TSP. For instance, an exact solution can be obtained easily using the state-of-the-art Concorde software ${ }^{2}$, while good solutions can be estimated for a reduced computational cost with optimized implementation of the Lin-Kernighan algorithms such as the LKH [18].

Oppositely, good parallel schedules are harder to obtain. While the problem expressed in Eq. (16) is closely related to the highly studied VRP, this formulation of the problem has, to our knowledge, no equivalent in the literature and has no available implementation. However, multiple efforts have been dedicated to the elaboration of efficient metaheuristic aiming to solve the VRP. Therefore, in order to solve the problems defined in Eq. (12) and Eq. (16), we implemented a genetic algorithm inspired by an approach derived from this literature.

\footnotetext{
${ }^{2}$ http://www.math.uwaterloo.ca/tsp/concorde/index.html
}

\subsection{Genetic algorithm}

Genetic algorithms [39] explore the solution space by simulating the evolution of a population of individuals. Each individual represents a solution to the optimization problem considered and has a fitness representing the goodness of its solution.

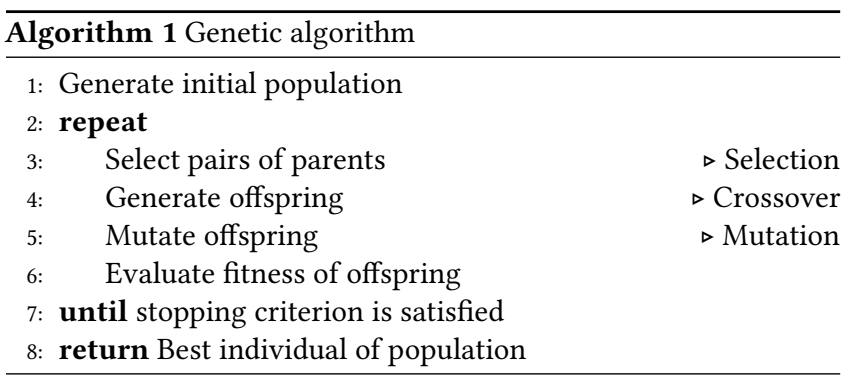

The population is initialized with individuals randomly generated. This initial population evolves by using genetic operators on individuals as represented in Algo. 1. The first operator, the crossover, consists in selecting two promising individuals that are then combined to form at least one offspring sharing characteristics of its parents. The second operator, the mutation, applies random alterations to each offspring. The offspring then replace the parent population. This scenario is repeated until a stopping criterion is reached such as the stagnation of the average population fitness.

The selection of parents, prior to the crossover, aims to promote the exploration of the solution space in the vicinity of the best individuals of the population. However, there is no guarantee that the best solution encountered is preserved through the evolution process. Therefore, elitism is employed to preserve the best individual unscathed and to maintain its presence in the next generation.

The described genetic algorithms were applied to both the sequential and parallel optimization problems. The fitness function was directly derived from the optimization problems defined in Eqs. (12) and (16). Given that good individuals should have higher fitness than worse ones, the computational cost of a schedule was inverted. Individuals represented thus the ordered sequence $\mathcal{R}$ for the sequential case and the ordered partitions $\mathcal{R}^{(\cdot)}$ for the parallel case. The selection strategies and the genetic operators employed for each implementation were specialized and are described hereafter.

\subsection{Algorithm for the sequential scheduling}

The selection of the parents was done using the well-known roulette selection that randomly selects individuals proportionally to their fitness. The crossover operator employed was the Order crossover [30, 34]. This operator mates two parents by selecting a contiguous subset of the ordering $\mathcal{R}$ of the first parent and then fills the missing elements by maintaining, as much as possible, the ordering expressed in the second parent. This approach aims to mix two good features expressed in the parents.

The mutation operator had two effects. First, it intensified the search in the individual vicinity by applying a simple local search algorithm [40]. This heuristic tries successively to apply a 2-opt, or 3-opt move, to the individual. These moves swap respectively two and three elements of $\mathcal{R}$ and were only kept when improving 
the individual fitness. Second, it diversified the search by applying random 2-opt moves that didn't necessarily improve its fitness.

\subsection{Algorithm for the parallel scheduling}

While the fitness of an individual is directly obtained from Eq. (16), this score only reflects the goodness of the costliest partitions $\mathcal{R}^{(\max )}$. Indeed, individuals sharing the same partitions $\mathcal{R}^{(\max )}$ have the same fitness while their overall cost (Eq. (15)) may be significantly different. This difference in overall cost can be however beneficial to an individual since it reflects a better partitioning.

The selection of individual was first based on the apparent individual fitness (Eq. (16)) and then on the overall cost (Eq. (15)). At this end, individuals were ranked by decreasing apparent fitness and ties were settled using their overall cost. Individuals were then selected with a probability proportional to their ranking.

The crossover operator was inspired by the Best Cost Crossover Route [31] originally designed for the VRP. A partition was randomly chosen from the first parent and its elements were removed from the partitioning $\mathcal{D}$ of the second parent. The removed elements were then reinserted using a greedy strategy consisting in finding the best possible insertion point for each element with respect to the overall cost.

As in the sequential scheduling, the mutation operator had several effects. The 2-opt heuristic was again used to optimize each partitions of an individual and 2- or 3-opt moves were used to mutate randomly an arbitrary partition. A third mutation was added to propose changes in the partitioning. This mutation removed one random element from an arbitrary partition and then defined the best possible insertion point in a second arbitrary partition.

Finally, given that this algorithm is employed whenever multiple processors are available for the gradient evaluation, parallel strategies for genetic algorithms [3,11] were employed. At the population level, up to 10 processors were used to evaluate the fitness of the population individuals. When more than 10 processors were available, this first strategy was combined with a multiple-population scheme to accelerate the convergence of the algorithm.

In the multiple-population scheme, each group of processors was responsible for the evolution of its own population. Some individuals of each population were then periodically exchanged with neighbouring populations. In our implementation, these migrants were selected according to experimental observations done by Bravo et al. [10]: a fixed number of the best and worst individuals of the source population were sent to a neighbouring population and replaced its worst individuals.

\section{EXPERIMENTAL EVALUATION}

In this section, we investigate the potential gains resulting from the scheduling of perturbations. First, we use synthetic DAGs having various graph properties to investigate the presented approach potential and to validate the theoretical results previously derived. Then, we apply this approach to evaluate gradients of a large scale application from the field of evolutionary biology aiming to detect positive selection in protein coding sequences [53].

\subsection{Experimental settings}

Our implementation is developed in $\mathrm{C}++$ and uses hybrid MPI/POSIX parallelism. The first set of experiments on synthetic DAGs were run on a cluster node composed of two Intel Xeon E5-2620V2 CPUs and $64 \mathrm{~GB}$ of RAM. Experiments on the large evolutionary biology application were conducted on a cluster composed of nodes having two Intel Xeon E5-2660V0 CPUs and 64 GB of RAM.

\subsection{Synthetic DAG}

Synthetic DAGs were simulated using a software generating random graphs for scheduling simulations [12]. This tool implements several methods that each generates graphs having a peculiar structure. Two methods were chosen to assess the effect of our approach: the Fan-in/Fan-out (FiFo) and the Layer-by-Layer (LbL) methods.

The FiFo method creates graphs by applying Fan-in and Fan-out phases. A Fan-in phase consists in the creation of a new vertex that have multiple parents in the DAG, while a Fan-out phase adds several newly created vertices to a child of an existing vertex in the DAG. The number of vertices created during each phase is controlled by user-specified values for the maximum inner degree $i d$ and the outer degree od of each vertex in the DAG.

The LbL method requires the user to specify the number $k$ of layers in which the vertices are assigned. Edges are then added with probability $p$ between two vertices of the DAG with an additional constraint on the layers. This constraint enables an edge to be created only if the parent vertex belongs to a layer $l_{i}$ that precedes the layer $l_{j}$ of the child vertex, such that $i<j$.

The FiFo method enables to control the graph structure. With $i d=o d=1$, this method generates linear graphs. If $o d$ is then increased while id is maintained, graphs generated are tree-like shaped and their longest path is inversely proportional to od. Increase in id creates a more complex graph structure and slowly augment the amount of edges in the graph. The LbL method offers a more direct control on the number of edges at the cost of a less specifiable structure. The edges number of graphs generated with this method is controlled by $p$, while the only structural control is attained by tuning $k$, the maximal size of the longest path.

Once graphs are simulated, arbitrary computational costs must be affected to their vertices. At this end, we experimented with various distribution for the vertices cost (e.g. uniform, bimodal, exponential). These experiments revealed that the scale of the performance gains resulting from our approach were consistent for individual type of graphs with cost simulated under different distributions. While no significant variations could be observed for the performance gains, the schedules obtained were however highly dependent on the distribution employed. Given that the use of synthetic DAGs aims at studying the improvements brought by scheduling, we chose to use an unitary cost for each vertex.

Theoretical performance bounds were previously identified during the definition of the optimization problems. These bounds were defined using assumptions on graphs properties such as their structure as well as the cost assigned to their vertices. Two measures made on the synthetic DAGs generated that are related to these properties are reported in table 1 . The first measure reported is the average size of the DAGs longest path. This value played a key role 
Table 1: Properties of the simulated DAGs. The Conf. column contains the parameters used for graphs simulation: $i d, o d$ for FiFo and $p$ for the LbL graphs. The parameter $k$ for $\mathrm{LbL}$ graphs was fixed as a fraction of $|V|$

\begin{tabular}{|c|c||c|r|r|r||r|r|r|r|}
\hline Type & Conf. & \multicolumn{3}{c||}{ Long. path size } & \multicolumn{4}{c|}{$C(\Psi)}$. \\
\hline \hline \multicolumn{2}{|c||}{$d=|V|$} & 20 & 50 & 100 & 200 & 20 & 50 & 100 & 200 \\
\hline \hline \multirow{3}{*}{ FiFo } & 1,1 & 20 & 50 & 100 & 200 & 11 & 26 & 51 & 101 \\
\cline { 2 - 10 } & 1,2 & 7.3 & 11.2 & 13.5 & 16.2 & 5.4 & 7.4 & 9 & 10.4 \\
\cline { 2 - 10 } & 1,4 & 7.2 & 8.5 & 11.5 & 13 & 5.1 & 6.4 & 7.5 & 8.6 \\
\hline \hline \multirow{3}{*}{ LbL } & 0.02 & 2 & 3.7 & 6 & 9 & 2 & 2.6 & 3.8 & 9.5 \\
\cline { 3 - 10 } & 0.05 & 2.8 & 5 & 8.3 & 16 & 2.5 & 4 & 11.2 & 42 \\
\cline { 3 - 10 } & 0.10 & 4.3 & 8.7 & 13.5 & 22.5 & 3.3 & 9.7 & 26.3 & 69.8 \\
\hline
\end{tabular}

when determining the worst-case computational cost of pairing two partial computations (Eq. (11)).

The second measure is the average cost of a partial computation $C(\Psi$. $)$ and is directly related to the cost $C(V)=|V|$ given that vertices have an unitary cost. This measure reflects the discrepancy between the computational cost of the DAG initialization $C(V)$ and the one of an ordering $C(R)$. The ratio between these two costs was shown to bound the performance gain resulting from the use of parallel computing resources (Eq. (17)).

5.2.1 Single processor experiments. We first assessed the gain coming from the use of partial computations for the gradient evaluations. This gain $G_{D}$ was measured as the ratio of the full cost required to compute $d+1$ functions and the cost of computing the sequence defined in Eq. (2). The average ratio, defined over 50 random orderings $\mathcal{R}$, is reported in table 2 . This result reveals a large variation of performance gains depending on the graphs type.

For FiFo graphs, apart for the case of linear graphs $(i d=o d=1)$, the performance gain increased proportionally with the graphs size $|V|$. Such gains were a direct consequence of the low amount of data dependencies expressed in this type of graph that enabled partial computations to significantly reduce the computational cost.

The gains reported for LbL graphs were less important due to the growing amount of dependencies that limited the potential of partial computations. Indeed, their average cost $C(\Psi$. $)$ reported in table 1 grew exponentially with the graphs size. While small graphs had few data dependencies and thus good performance gains, large graphs had a significant amount of data dependencies and their average cost $C(\Psi$.$) grew up to 35 \%$ making thus the use of partial computations less effective on such graphs.

The gains brought by scheduling the partial computations was then assessed by measuring the cumulative gain $G_{S}$ identifying the speedup over gradients evaluation using random schedules. Performance gains obtained on FiFo graphs highlighted different aspects. First, good schedules for linear graphs provided a gain of $\approx 32 \%$ over the random schedules, regardless of the graphs size. This trend shifted as soon as the outer degree of vertices increased. For graphs having $o d=2$, the performance gain started from $\approx 25 \%$ and increased with the graph size. A similar trend could be observed for graph having od $=4$ with, however, slightly lower gains.

These trends reveal that the depth of the graph has no importance for linear graphs $(i d=o d=1)$, but directly impacts the quality of
Table 2: Performance gains on synthetic graphs. $G_{D}$ defines the speedup gained by using partial computation with random schedules. $G_{S}$ represents further speedups achieved by scheduling. The final accelerations are then given as $G_{D} \times G_{S}$

\begin{tabular}{|c|c|c|c|r|c|r|r|r|r|}
\hline \multirow{3}{*}{ Type } & \multirow{2}{*}{ Conf. } & \multicolumn{8}{|c|}{ Size $(d=|V|)$} \\
\cline { 3 - 10 } & & \multicolumn{2}{|c}{20} & \multicolumn{2}{|c|}{50} & \multicolumn{2}{|c|}{100} & \multicolumn{2}{|c|}{200} \\
\cline { 3 - 10 } & $G_{D}$ & $G_{S}$ & $G_{D}$ & $G_{S}$ & $G_{D}$ & $G_{S}$ & $G_{D}$ & $G_{S}$ \\
\hline \hline \multirow{3}{*}{ FiFo } & 1,1 & 1.43 & $\mathbf{1 . 3 2}$ & 1.44 & $\mathbf{1 . 3 4}$ & 1.44 & $\mathbf{1 . 3 2}$ & 1.45 & $\mathbf{1 . 3 3}$ \\
\cline { 2 - 10 } & 1,2 & 2.67 & $\mathbf{1 . 2 6}$ & 4.69 & $\mathbf{1 . 3 3}$ & 7.34 & $\mathbf{1 . 3 8}$ & 11.82 & $\mathbf{1 . 4 3}$ \\
\cline { 2 - 10 } & 1,4 & 2.89 & $\mathbf{1 . 2 4}$ & 5.39 & $\mathbf{1 . 2 8}$ & 8.41 & $\mathbf{1 . 3 8}$ & 15.02 & $\mathbf{1 . 3 9}$ \\
\hline \hline \multirow{3}{*}{ LbL } & 0.02 & 5.50 & $\mathbf{1 . 1 4}$ & 10.45 & $\mathbf{1 . 2 0}$ & 14.71 & $\mathbf{1 . 3 0}$ & 11.64 & $\mathbf{1 . 4 4}$ \\
\cline { 2 - 10 } & 0.05 & 4.64 & $\mathbf{1 . 2 2}$ & 6.79 & $\mathbf{1 . 3 3}$ & 5.27 & $\mathbf{1 . 4 4}$ & 3.17 & $\mathbf{1 . 4 7}$ \\
\cline { 2 - 10 } & 0.10 & 3.63 & $\mathbf{1 . 2 9}$ & 3.32 & $\mathbf{1 . 4 0}$ & 2.55 & $\mathbf{1 . 4 1}$ & 1.97 & $\mathbf{1 . 3 9}$ \\
\hline
\end{tabular}

orderings $\mathcal{R}$ for graphs having multiple branches $(i d=1, o d>1)$. Under such context, orderings containing jumps from separated branches of the graph induced unnecessary computations. As the number of branches increased ( $o d \gg 1$ ), the depth of the tree decreased, and thus the detrimental effect of jumps diminished. This trend is clearly observable in the extreme case where the graph would only have the root as non-terminal vertex. Under this condition, the maximal depth would be equal to one and thus orderings of the partial computations would not affect the performance.

Similar increases in performance relative to an augmentation in graphs size were observed on LbL graphs. For such graphs, an increase in the number of edges $(p=0.02 \rightarrow p=0.05)$ between layers also conducted to an increase of performance when proper scheduling of the computations where used. However, as the inner and outer degrees of each vertices increased ( $p=0.05 \rightarrow p=0.10$ ), the utility of scheduling became less rewarding. Indeed, as the data dependencies in the graph grew, the average cost of partial computation converged toward the full evaluation $\operatorname{cost} C(\mathcal{R})$.

In summary, the difficulty of obtaining good schedules is directly related with the structure of the graphs. Structures characterized by multiple deep unconnected branches may lead to a high amount of unnecessary computations that can be avoided by the use of proper computations schedules. On the opposite, graphs having a large amount of data dependencies are less prone to benefit from partial computation as well as their ordering.

Nonetheless, our experiments revealed that the use of scheduled partial computations always accelerated the gradient evaluation by at least a twofold factor in the worst case and as much as a twentyfold factor in the best cases ${ }^{3}$ when compared to the time required to evaluate the $(d+1)$ functions without partial computations. More importantly, for graphs having few data dependencies and several branches (e.g. FiFo with $i d=1$, od $>1$ ), the function and gradient evaluations costed $\frac{d}{G_{D} \cdot G_{S}} \approx 10$ times the cost of the original function evaluation independently of the graph size.

5.2.2 Multiprocessors experiments. We first assessed the quality of the schedules obtained from the genetic algorithm for the optimization problem defined in Eq. (16). We measured the speedup of the parallel evaluation of the gradient as the execution time of the

${ }^{3}$ E.g. FiFo with $d=200, i d=1$, od $=4 \rightarrow G_{D} \cdot G_{S}=20.88$ and LbL with $d=100, p=0.02 \rightarrow G_{D} \cdot G_{S}=19.12$. 
best sequential schedule divided by the observed parallel execution time. This speedup isolates the acceleration gain resulting from the use of multiple processors. However, it is important to note that the absolute gain resulting from the use of partial computations on multiprocessors is the product of the gains $G_{D}, G_{S}$ as well as the speedup measured in the upcoming experiments.

In order to give a point of comparison, we derived two approximate speedups using the ratio $C(\mathcal{R}) / C\left(\mathcal{R}^{(\cdot)}\right)$ and Eq. (17). The first approximate speedup represents the best one and thus use for $C(\mathcal{R})$ the best observed sequential schedule. The second one identifies the worst possible speedup, in term of ordering, and thus use the average $C(\mathcal{R})$ measured over several random sequential schedules.

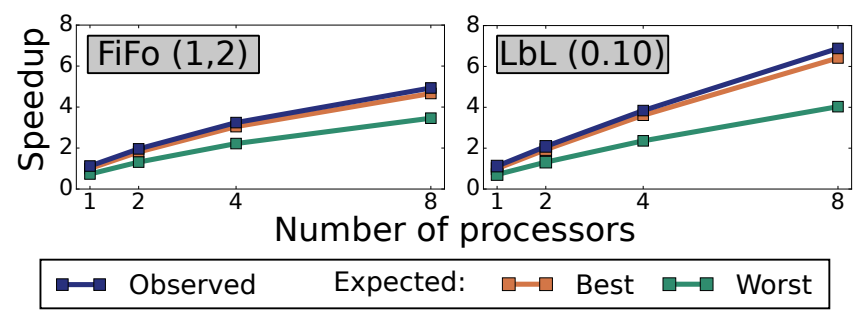

Figure 1: Speedups for FiFo and LbL graphs with $|V|=100$. The Observed speedups for the gradient evaluations are compared with the expected Best and Worst theoretic speedups.

For both type of graphs, the measures showed in Fig. 1 confirmed the validity of the approximations based on Eq. (17). Measured speedups revealed that the schedules obtained significantly surpassed the worst expected speedup and even slightly surpassed the best one. This second result may seem surprising, but can be readily explained. First, the strict triangle inequality property of the cost function ensures that the partition of the best obtained sequential schedule results in any case in a reduction of the computational cost. Second, the cost of the first partial computation on processors $p>1$ is included in the initialization $\operatorname{cost} C(V)$ which may absorb some of the most costliest partial computation (Eq. (14)).

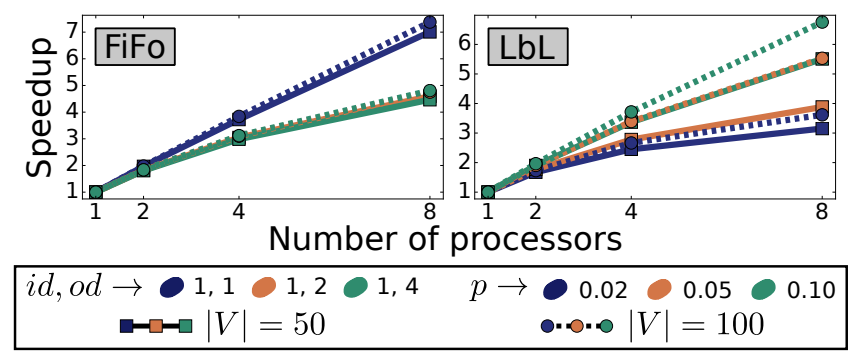

Figure 2: Speedup for FiFo and LbL graphs

A more thorough study of the results shown in Fig. 2 confirmed that the ratio between the total cost of $C(\mathcal{R})$ and the initialization cost $C(V)$ is a key limitation identified (see Eq.(18)). Therefore to achieve notable speedups with multiple processors, the amount of parallel computations contained in $C(\mathcal{R})$ must be significantly bigger than the sequential irreducible computational cost $C(V)$.

Table 1 displays the average cost per partial computation of the DAG $\Psi_{(\cdot)}$ which is directly related to the total cost $C(\mathcal{R})$. FiFo graphs had a nearly linear growth in $\left.\Psi_{(.)}\right)$as a function of the graph size. Therefore, their measured speedup remained nearly invariant to the graphs size. On the other hand, the average cost $\Psi_{(\cdot)}$ of LbL graphs augmented more sharply as a function their size; LbL graphs were subject to a significant increase in speedup as the graphs size grew. A similar trend could be observed with the growth of the parameter $p$ used in LbL graphs simulation that translated in more computational dependencies, or edges, existing in the graph.

In conclusion, graphs for which the sequential scheduling had the smallest effect were more prone to benefit from parallel computing resources. For example, for LbL graphs with $d=100, p=0.05$ and $p=0.10$, gradients were evaluated up to 5.5 and 6.9 times faster with 8 processors than their sequential evaluation with the best known schedule. These gains amounted to a total speedup of 42 and 25 when compared to the sequential evaluation time without partial computations. In the best scenarios, the evaluation cost of the function and the gradient with 8 processors amounted to approximately twice the cost of a sequential function evaluation.

5.2.3 Validation of the schedules. We validated the schedules obtained using the genetic algorithms on linear graphs generated with the FiFo method ( $i d=o d=1)$. For these graphs, the best sequential schedule is known and represents the sequence of nodes visited in a top-down traversal. To validate the scheduling method, the similarity between these optimal schedules and the computed ones was measured as $\left(1-\frac{c_{c}-c_{b}}{c_{w}-c_{b}}\right) \in[0 . .1]$, with $c_{c}, c_{b}$ and $c_{w}=2 c_{b}$ (Eq.(11)) being the cost of the computed, best and worst schedules. Similarity scores for parallel schedules were estimated using $c_{b}$ as the best expected schedule (Eq (17)).

We report hereafter the average similarity and relative standard deviation (RSD) over 20 schedules for different settings. In sequential, the similarity measured was of $0.998 \pm 0.06 \%$ for graphs having $|V|=100$ vertex and $0.993 \pm 0.07 \%$ for $|V|=500$. In the parallel setting, with 8 and 16 processors, the similarity was observed at $1 \pm 0.06 \%$ for graphs having $|V|=500$. Similar results were obtained with various settings; validating thus the accuracy and robustness of our genetic algorithms at obtaining good schedules.

\subsection{An evolutionary biology application}

The final experiment employs the strategy presented in this article on an evolutionary biology application: the detection of positive selection on protein coding sequences. Using molecular sequences for multiple species having a predefined phylogenetic tree, positive selection is inferred using statistical analyses based on the maximum likelihood estimation method. Since the parameters of such likelihood function are continuous, the optimization methods employed for its maximization are gradient-based and thus require a large number of gradient evaluations.

Several models for the detection of positive selection exist. We selected two of the most widely used models and employed their respective implementation as reference for the performance measures. The first model [53] infers the proportion of three global scenarios of molecular evolution over the phylogeny and has been recently 
implemented in a state-of-the-art software: FastCodeML [45]. The second model [32] infers the same three scenarios of evolution; however, separately for each node of the (phylogenetic) tree. This model is only implemented in the HYPHY software [33].

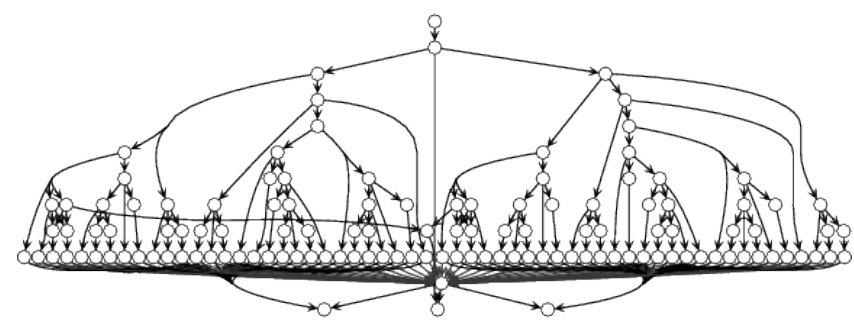

Figure 3: Example of a directed task graph for the evolutionary biology model based on a dataset having 16 species

The likelihood function of each model leads to significantly different DAG structures. DAGs resulting from the first model contain two tree-structure reflecting the phylogeny; each depending on a vertex set dedicated to a global evolutionary scenario. The vertex set assigned to the third scenario is linked to the tree nodes designed as evolving under positive selection. Such a DAG can be observed in Fig. 3 for a dataset with 16 species. The second model generates DAGs having a single tree-structure with each tree node having three vertex sets; one for each local evolutionary scenario.

For the experiment, three different settings of our implementation were considered. First, we measured the execution times of gradient evaluations using sequential partial computations with random schedules such as to isolate the effect of partial computations. Second, we used the genetic algorithms to define good sequential and parallel schedules such as to assess their impact. Finally, we combined the parallel evaluations of gradient presented in this article with parallel function evaluations. This mixed parallel computations approach is straightforward to implement since each partial computation represents a subset of the full DAG and can be directly scheduled on multiple processors using any kind of static or dynamic load balancing approach [23].

The execution time of these settings were mainly measured on two large datasets; one for each model. The dataset DS1 employed for the first model counted more than 1,000 species and 250 codons ${ }^{4}$; resulting in a model with close to $d \approx 2000$ input variables. The second dataset DS2 had more than 500 species, 500 codons and $d \approx 6000$ input variables. DAGs for the likelihood function of both models consisted in approximately $|V| \approx 8000$ vertex.

5.3.1 Performance comparison with FastCodeML. In a sequential setting, our implementation significantly outperformed FastCodeML on datasets of various size, as shown in Fig. 4. This speedup results from two improvements. First, FastCodeML scarcely employs partial computations. The use of partial computations in our implementation leads to a speedup of approximately $\frac{n \text { Species }}{\log (\text { nspecies })}$

${ }^{4}$ Codons are nucleotide triplet coding for amino acids and forming a 61 symbols alphabet. due to the DAG structure. Second, our implementation uses matrixmatrix (level 3 BLAS) operations while FastCodeML uses matrixvector (level 2 BLAS) operations. The first improvement explains the increase in speedup with the species number, while the second one explains the increase in speedup with the number of codons.

On the large dataset DS1, our implementation was approximately 400 times faster than FastCodeML when a single processor was used (Fig. 5a). Using a schedule obtained from the genetic algorithm contributed to a 1.62 speedup when compared to the use of random schedules. Parallel gradient evaluations with good schedules further increased the speedup as more processors were employed and accelerated upto 11,000 times the gradient evaluations when compared to FastCodeML (Fig. 5b). Furthermore, the combined use of parallel gradient and function evaluations showed an increase in parallel efficiency when compared to the sole use of parallel gradient evaluations as the processors number increased.

This increase in efficiency can be explained by the upper performance bound limiting the parallel gradient evaluation (Eq. (18)). Indeed, our approach is limited by the ratio of the total cost $C(\mathcal{R})$ and the DAG initialization cost $C(V)$. When using parallel functions evaluation the execution time of both costs is reduced as a function of the amount of processors used. However, the computation included in $C(V)$ is benefiting more from parallel function evaluations because the full DAG computation represents a larger potential for parallel computation than from partial computations that are included in the cost $C(\mathcal{R})$. Therefore, when parallel function evaluations are employed, the execution time associated with the cost $C(V)$ decreases faster than the one of $C(\mathcal{R})$, which induces an increase in the upper performance bound.

5.3.2 Performance comparison with HYPHY. The comparison of our implementation to HYPHY on the large dataset DS2 led to similar trends in performance gains, although to a lesser extent. In a sequential setting, our implementation was only 1.7 times faster than HYPHY (Fig. 5c). This less than stellar increase in performance gain is explained by the use of partial computations in HYPHY. Indeed, both implementations only differ in the DAG structure and the scheduling of partial computations. Gradient evaluations with random schedules and our implementation were slightly faster $(1.24 \times)$ than HYPHY. A subsequent speedup of 1.36 was observed when schedules resulting from the genetic algorithm were employed.

When using multiple processors for parallel $f(X)$ evaluations, the performance of HYPHY and our implementation were consistent (Fig. 5c). However, parallel gradient evaluations as well as mixed parallel evaluations enabled the scaling of our implementation to significantly outperform HYPHY (Fig. 5c and d). Indeed, our implementation reached up to a 66 speedup with 256 processors, while HYPHY stalled to 3.1 with 8 processors.

5.3.3 Assessing the schedules quality. We report hereafter average schedule scores and their relative standard deviations for schedules obtained for the first model with dataset DS1. Random schedules for $P=1$ processor had a score of $45.5 \pm 2.7 \%$, while computed schedules had a score of $30.1 \pm 0.8 \%$. With $P=16$ and $P=32$, the score improved to $3.36 \pm 0.7 \%$ and $2.3 \pm 0.1 \%$ respectively.

The accuracy of the genetic algorithm is hard to assess without knowing the optimal schedule for this experiment. However, the improved score of the sequential schedules compared to the one of 


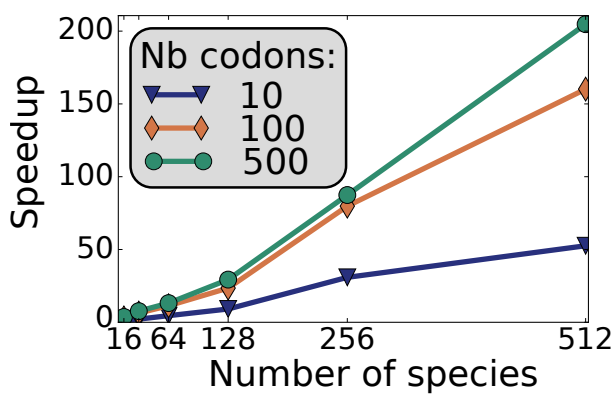

Figure 4: Speedup for sequential executions of our implementation against FastCodeML on datasets of various sizes.

the random schedules $(1.5 x)$ gives an insight on the schedules quality. Furthermore, the small interval in which the schedules scores fitted for each setting confirmed the robustness of the algorithm.

5.3.4 Performance gains and scalability summary. Since the previous experiments only reported performance gains relative to the reference implementations, we summarize these results with other metrics to emphasize their implications.

- FastCodeML took nearly 3 hours to evaluate $f(X)$ and $\nabla f(X)$ on DS1. Our approach reduced this time to $\approx 30$ seconds with $P=1$ and $\approx 1$ second with $P=256$.

- Evaluating gradients according to Eq. (1) requires $O(d)$ evaluations of $f(X)$. Defining $T_{f}$ as the sequential evaluation time for $f(X)$ with our approach enables its direct comparison with the naive $d \times T_{f}$. With $P=1$, the evaluation time for $f(X)$ plus $\nabla f(X)$ for the first and second models amounted to $24.6 T_{f}$ and $28.6 T_{f}$, resp. It reduced to $1.2 C_{f}$ and $1.4 f C_{f}$, resp. with $P=64$ and further decreased to $0.6 C_{f}$ and $0.9 C_{f}$, resp. with $P=256$.

- Our implementation significantly outperformed the scaling of HYPHY. This software scaling stalled with $P>8$ while performance gains were still observed with our approach and $P>200$ thanks to the parallel gradient and mixed parallel evaluations.

- The parallel efficiency relative to our sequential implementation lowered as $P$ increased. A $100 \%$ efficiency was achieved with $P \leq 8$, lowered to $50 \%$ with $P \approx 20$ and reached $20 \%$ with $P \approx 100$.

5.3.5 Memory footprints. The memory used is by a software is highly dependent on implementation choices. However, memory footprints should be consistent for the implementations studied given that the size of the dataset grows linearly with the codons and species numbers. Using partial computations, as presented in this article, further increased the minimal footprints up to a small multiplicative factor $(\approx 2)$.

The memory usage measured for the first model with the dataset DS1 was close to 1.5GB for FastCodeML and 1GB for our implementation. Smaller memory footprints were measured on the second model with dataset DS2: HYPHY only used 350MB while our implementation used 500MB. Since the datasets have roughly the same size ( $n$ Codons $\times n$ Species), the difference in the memory footprint

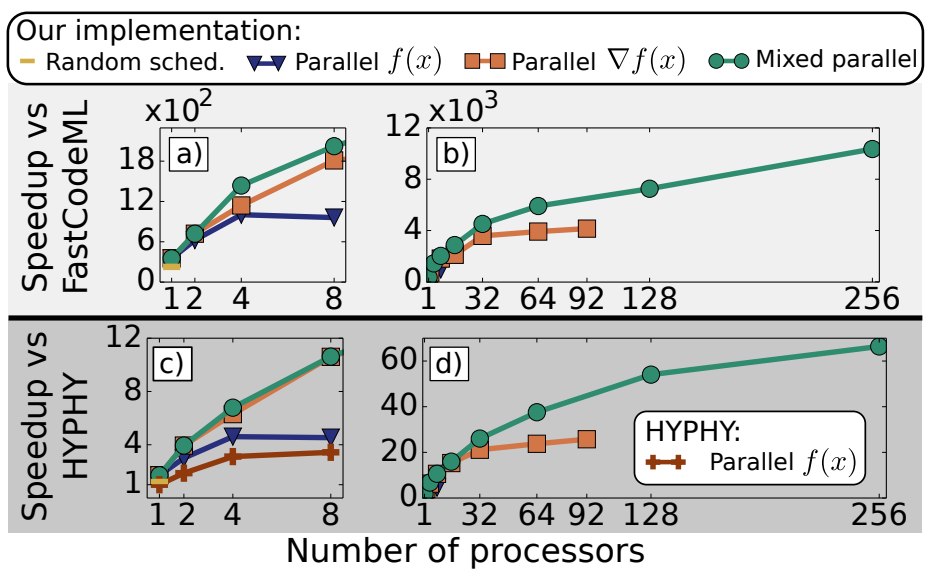

Figure 5: Speedup against FastCodeML and HYPHY.

between both models can be explained by their DAGs structure; the first model has two copies of the phylogenetic tree in its DAG while the second has only once such structure.

While these memory footprints were not a limiting factor during these experiments, larger existing datasets [35] or more complex models [36] would consume significantly more memory. For instance, the amphibian phylogeny [35] with 4600 species and 800 codons would require close to $15 \mathrm{~GB}$ of memory per DAG instance.

\section{CONCLUSION AND PERSPECTIVES}

We presented a method enabling efficient gradient evaluations for large scale applications having their function modeled as DAG. The performance gains measured over a naive use of the finite difference method resulted from the joint effect of partial computations and their scheduling on one or multiple processors. These sequential and parallel scheduling problems were formalized as discrete optimization problems and some of their properties were derived.

The performance of the presented approach was characterized on several structure of DAGs. The efficiency of the gradient evaluations was shown to be consistently improved for synthetic DAGs and significant gains were observed when employing scheduling approaches for single or multiprocessor architectures. Finally, this method was employed for a real large scale application from the field of evolutionary biology. On two different experiments, the gradient evaluations were significantly speed up when compared to state-of-the-art implementations. When 256 processors were employed, $f(X)$ and $\nabla(X)$ were jointly evaluated in less time than required to evaluate $f(X)$ in sequential.

This efficient approach for the gradient evaluation can be further improved. For instance, while the effort of obtaining good schedules is generally insignificant compared to numerous gradient evaluations, heuristics designed for specific DAG structure or more general one could be designed to further reduce this computational effort. The strict triangle inequality property (Thm. 3.1) could serve as good starting point for the elaboration of such methods. Another potential improvement would be to employ more advanced scheduling strategies such as the task-duplication scheduling [2] and to consider the joint scheduling of parallel function 
and gradient evaluations. Such approach would aim to increase the parallel efficiency by finding the best schedule for partial computations, while minimizing the load unbalance caused by partial computations having poor potential for parallel evaluation.

In conclusion, after a thorough investigation, we showed that evaluating gradients with the finite difference method can be efficiently conducted for large scale applications when their function is expressed as a DAG. The presented method is sufficiently generic to account for other variants of the finite difference method such as the center-difference formula aiming to augment the gradient accuracy. Furthermore, thanks to the use of DAGs, parallel function evaluations can be integrated without further changes and are shown to increase the parallel efficiency. All these combined advantages makes this simple, yet efficient, approach a significant asset for large scale applications requiring gradient evaluations.

\section{ACKNOWLEDGMENTS}

We thank the anonymous reviewers for their careful reading of our manuscript and their many insightful comments and suggestions. We also thank the Center of Advanced Modeling of Sciences and the University of Geneva HPC team for providing the computational resources for this study. This work was supported by the Swiss National Science Foundation under grant CR32I3_143768.

\section{REFERENCES}

[1] Kunal Agrawal, Jing Li, Kefu Lu, and Benjamin Moseley. 2016. Scheduling parallel DAG jobs online to minimize average flow time. In Proceedings of the Twenty-Seventh Annual ACM-SIAM Symposium on Discrete Algorithms. SIAM, 176-189.

[2] Ishfaq Ahmad and Yu-Kwong Kwok. 1998. On Exploiting Task Duplication in Parallel Program Scheduling. IEEE Trans. Parallel Distrib. Syst. 9, 9 (Sept. 1998), 872-892. https://doi.org/10.1109/71.722221

[3] Enrique Alba and José M. Troya. 1999. A survey of parallel distributed genetic algorithms. Complexity 4, 4 (mar 1999), 31-52.

[4] David L Applegate, Robert E Bixby, Vasek Chvatal, and William J Cook. 2011. The traveling salesman problem: a computational study. Princeton university press.

[5] Michael Bartholomew-Biggs, Steven Brown, Bruce Christianson, and Laurence Dixon. 2000. Automatic differentiation of algorithms. F. Comput. Appl. Math 124, 1-2 (dec 2000), 171-190. https://doi.org/10.1016/S0377-0427(00)00422-2

[6] Tolga Bektas. 2006. The multiple traveling salesman problem: an overview of formulations and solution procedures. Omega 34, 3 (jun 2006), 209-219. https://doi.org/10.1016/j.omega.2004.10.004

[7] C. H. Bennett. 1973. Logical Reversibility of Computation. IBM f. Res. Dev. 17, 6 (Nov. 1973), 525-532. https://doi.org/10.1147/rd.176.0525

[8] Christian H. Bischof, H. Martin Bücker, and Arno Rasch. 2010. Enabling Technologies for Robust High-Performance Simulations in Computational Fluid Dynamics. Springer Berlin Heidelberg, Berlin, Heidelberg, 153-180. https: //doi.org/10.1007/978-3-642-04088-7_7

[9] Erin N. Bodine, Suzanne Lenhart, and Louis J. Gross. 2014. Mathematics for the Life Sciences. (aug 2014).

[10] Yesnier Bravo, Gabriel Luque, and Enrique Alba. 2013. Migrants Selection and Replacement in Distributed Evolutionary Algorithms for Dynamic Optimization. 217 (2013), 155-162. DOI: 10.1007/978-3-319-00551-5_19.

[11] Erick Cantú-Paz. 1998. A survey of parallel genetic algorithms. Calculateurs paralleles, reseaux et systems repartis 10, 2 (1998), 141-171.

[12] Daniel Cordeiro, Grégory Mounié, Swann Perarnau, Denis Trystram, Jean-Marc Vincent, and Frédéric Wagner. 2010. Random Graph Generation for Scheduling Simulations. (2010), 60:1-60:10.

[13] Benjamin Dauvergne and Laurent Hascoët. 2006. The data-flow equations of checkpointing in reverse automatic differentiation. In International Conference on Computational Science. Springer, 566-573.

[14] Andreas Griewank and Andrea Walther. 2008. Evaluating derivatives: principles and techniques of algorithmic differentiation. Siam.

[15] L. Hascoët and V. Pascual. 2013. The Tapenade Automatic Differentiation tool: Principles, Model, and Specification. ACM Trans. Math. Software 39, 3 (2013), 20:1-20:43
[16] Laurent Hascoët and Ala Taftaf. 2016. On the correct application of AD checkpointing to adjoint MPI-parallel programs. Research Report RR-8864. Inria Sophia Antipolis. https://hal.inria.fr/hal-01277449

[17] Ibrahim Abaker Targio Hashem, Ibrar Yaqoob, Nor Badrul Anuar, Salimah Mokhtar, Abdullah Gani, and Samee Ullah Khan. 2015. The rise of "big data" on cloud computing: Review and open research issues. Information Systems 47 (jan 2015), 98-115.

[18] Keld Helsgaun. 2000. An effective implementation of the Lin-Kernighan traveling salesman heuristic. European fournal of Operational Research 126, 1 (2000), 106130 .

[19] Sebastian Höhna, Tracy A. Heath, Bastien Boussau, Michael J. Landis, Fredrik Ronquist, and John P. Huelsenbeck. 2014. Probabilistic Graphical Model Representation in Phylogenetics. Systematic Biology 63, 5 (sep 2014), 753-771.

[20] Michael I. Jordan. 2004. Graphical Models. Statist. Sci. 19, 1 (feb 2004), 140-155.

[21] Slawomir Koziel, Leifur Leifsson, Michael Lees, Valeria V.Krzhizhanovskaya, Jack Dongarra, Peter M.A. Sloot, Markus Towara, Michel Schanen, and Uwe Naumann. 2015. International Conference On Computational Science, ICCS 2015MPI-Parallel Discrete Adjoint OpenFOAM. Procedia Computer Science 51 (jan 2015), 19-28.

[22] Suresh Nanda Kumar and Ramasamy Panneerselvam. 2012. A survey on the vehicle routing problem and its variants. Intelligent Information Management 4, 3 (2012), 66.

[23] Yu-Kwong Kwok and Ishfaq Ahmad. 1999. Static Scheduling Algorithms for Allocating Directed Task Graphs to Multiprocessors. ACM Comput. Surv. 31, 4 (dec 1999), 406-471.

[24] Joaquim Martins, John Hwang, John Hwang, and John Hwang. 2013. Review and Unification of Methods for Computing Derivatives of Multidisciplinary Computational Models. AIAA fournal 51, 11 (2013), 2582-2599.

[25] Vivien Marx. 2013. Biology: The big challenges of big data. Nature 498, 7453 (jun 2013), 255-260. https://doi.org/10.1038/498255a

[26] Uwe Naumann. 2012. The Art of Differentiating Computer Programs: An Introduction to Algorithmic Differentiation. 24 (2012).

[27] Uwe Naumann, Laurent Hascoët, Chris Hill, Paul Hovland, Jan Riehme, and Jean Utke. 2008. A Framework for Proving Correctness of Adjoint Message-Passing Programs. (2008), 316-321.

[28] Rasmus Nielsen and Ziheng Yang. 1998. Likelihood models for detecting positively selected amino acid sites and applications to the HIV-1 envelope gene. Genetics 148, 3 (1998), 929-936.

[29] Jorge Nocedal and Stephen J. Wright. 2006. Numerical optimization. (2006).

[30] IM Oliver, DJd Smith, and John RC Holland. 1987. Study of permutation crossover operators on the traveling salesman problem. In Genetic algorithms and their applications: proceedings of the second International Conference on Genetic Algorithms: July 28-31, 1987 at the Massachusetts Institute of Technology, Cambridge, MA. Hillsdale, NJ: L. Erlhaum Associates, 1987.

[31] Beatrice Ombuki-Berman and Franklin T. Hanshar. 2009. Using Genetic Algorithms for Multi-depot Vehicle Routing. 161 (2009), 77-99.

[32] Sergei L. Kosakovsky Pond, Ben Murrell, Mathieu Fourment, Simon D. W. Frost, Wayne Delport, and Konrad Scheffler. 2011. A Random Effects Branch-Site Model for Detecting Episodic Diversifying Selection. Molecular Biology and Evolution 28, 11 (Nov. 2011), 3033-3043. https://doi.org/10.1093/molbev/msr125

[33] Sergei L Kosakovsky Pond and Spencer V Muse. 2005. HyPhy: hypothesis testing using phylogenies. In Statistical methods in molecular evolution. Springer, 125181.

[34] Jean-Yves Potvin. 1996. Genetic algorithms for the traveling salesman problem. Annals of Operations Research 63, 3 (jun 1996), 337-370.

[35] R. Alexander Pyron, Frank T. Burbrink, and John J. Wiens. 2013. A phylogeny and revised classification of Squamata, including 4161 species of lizards and snakes. BMC Evolutionary Biology 13 (2013), 93. https://doi.org/10.1186/1471-2148-13-93

[36] Le Si Quang, Olivier Gascuel, and Nicolas Lartillot. 2008. Empirical profile mixture models for phylogenetic reconstruction. Bioinformatics 24, 20 (2008), 2317-2323.

[37] Louis B Rall and George F Corliss. 1996. An introduction to automatic differentiation. Computational Differentiation: Techniques, Applications, and Tools (1996), $1-17$.

[38] Arno Rasch, H Martin Bucker, and Christian H Bischof. 2008. Automatic computation of sensitivities for a parallel aerodynamic simulation. Parallel Computing: Architectures, Algorithms and Applications. Advances in Parallel Computing 15 (2008), 303-310.

[39] Colin R. Reeves. 2010. Genetic Algorithms. 146 (2010), 109-139. DOI: 10.1007/9781-4419-1665-5_5.

[40] César Rego, Dorabela Gamboa, Fred Glover, and Colin Osterman. 2011. Traveling salesman problem heuristics: Leading methods implementations and latest advances. European fournal of Operational Research 211, 3 (jun 2011), 427-441. https://doi.org/10.1016/j.ejor.2010.09.010

[41] Sartaj K. Sahni. 1976. Algorithms for Scheduling Independent Tasks. F. ACM 23, 1 (jan 1976), 116-127. 
[42] Michel Schanen, Uwe Naumann, Laurent Hascoët, and Jean Utke. 2010. ICCS 2010 Interpretative adjoints for numerical simulation codes using MPI. Procedia Computer Science 1, 1 (2010), 1825 - 1833.

[43] Zachary D. Stephens, Skylar Y. Lee, Faraz Faghri, Roy H. Campbell, Chengxiang Zhai, Miles J. Efron, Ravishankar Iyer, Michael C. Schatz, Saurabh Sinha, and Gene E. Robinson. 2015. Big Data: Astronomical or Genomical? PLoS Biol 13, 7 (jul 2015), e1002195. https://doi.org/10.1371/journal.pbio.1002195

[44] Jean Utke, Laurent Hascoët, Patrick Heimbach, Chris Hill, Paul Hovland, and Uwe Naumann. 2009. Toward adjoinable MPI. In Parallel \& Distributed Processing, 2009. IPDPS 2009. IEEE International Symposium on. IEEE, 1-8.

[45] Mario Valle, Hannes Schabauer, Christoph Pacher, Heinz Stockinger, Alexandros Stamatakis, Marc Robinson-Rechavi, and Nicolas Salamin. 2014. Optimization strategies for fast detection of positive selection on phylogenetic trees. Bioinformatics (jan 2014), btt760. https://doi.org/10.1093/bioinformatics/btt760

[46] C. Voglis, P. E. Hadjidoukas, I. E. Lagaris, and D. G. Papageorgiou. 2009. A numerical differentiation library exploiting parallel architectures. Computer Physics Communications 180, 8 (aug 2009), 1404-1415. https://doi.org/10.1016/j. cpc.2009.02.004

[47] Yu. M. Volin and G. M. Ostrovskii. 1985. Automatic computation of derivatives with the use of the multilevel differentiating technique-1. Algorithmic basis. Computers \& Mathematics with Applications 11 (nov 1985), 1099-1114.

[48] Martin J. Wainwright and Michael I. Jordan. 2007. Graphical Models, Exponentia Families, and Variational Inference. Foundations and Trend $s \circledR$ in Machine Learning 1, 1-2 (2007), 1-305. https://doi.org/10.1561/2200000001

[49] Andrea Walther and Andreas Griewank. Getting Started with ADOL-C.. In Combinatorial scientific computing. 181-202.

[50] Wei Xu, Xi Chen, and Thomas F Coleman. 2014. The efficient application of automatic differentiation for computing gradients in financial applications. $\mathcal{F}$ Comput. Finance (2014). name number (url).

[51] Ziheng Yang. 1993. PAML: Phylogenetic Analysis by Maximum Likelihood. Molecular Biology and Evolution 24 (1993), 1586-1591.

[52] Ziheng Yang. 2007. PAML 4: phylogenetic analysis by maximum likelihood. Molecular biology and evolution 24, 8 (2007), 1586-1591.

[53] Ziheng Yang and Rasmus Nielsen. 2002. Codon-Substitution Models for Detecting Molecular Adaptation at Individual Sites Along Specific Lineages. Molecular Biology and Evolution 19, 6 (jun 2002), 908-917. 